\title{
First case of congenital idiopathic hypohidrosis in China*
}

\author{
$\mathrm{Ge} \mathrm{Shi}^{1}$ \\ Ying Zhou ${ }^{1}$ \\ Yi-Ming Fan ${ }^{1}$
}

\author{
Cheng-Yao $\mathrm{Zhu}^{1}$ \\ Yan-Ping Yang ${ }^{1}$
}

DOI: http://dx.doi.org/10.1590/abd1806-4841.20153327

\begin{abstract}
A 43-year-old Chinese man presented with generalized hypohidrosis, which he had had since birth, without obvious abnormalities of other skin appendages except a sparse beard and axillary hairs. The sweat test revealed localized sweating on the face, axillae and palms. Histopathologic examination showed that the sweat glands were absent in the forearm and thigh, but some eccrine and apocrine sweat glands were present in the right axilla. S-100 was expressed in the nerve terminals surrounding the acini and ducts of the eccrine sweat glands, while PGP9.5 was positive in the acini of apocrine glands and the nerve terminals surrounding the eccrine glands in the axilla. To our knowledge, this is the first case of congenital idiopathic hypohidrosis in China.
\end{abstract}

Keywords: Hypohidrosis; Pathology; Sweat glands

\section{INTRODUCTION}

Generalized anhidrosis is an uncommon, congenital or acquired problem characterized by heat intolerance and nonsweating. ${ }^{1}$ Idiopathic, simple, or isolated anhidrosis refers to generalized anhidrosis without any associated problems. ${ }^{2,3}$ To date, 32 cases of idiopathic anhidrosis have been reported in the English and Japanese literatures but there have only been 6 patients with congenital idiopathic anhidrosis in whom skin biopsy showed complete absence, dysplasia or atrophy of sweat glands. ${ }^{2,4-6}$ However, no similar disorders have been documented in the China National Knowledge Infrastructure (CNKI) and Wanfang database. The authors describe herein the first case of congenital idiopathic hypohidrosis in China.

\section{CASE REPORT}

A 43-year-old Chinese man presented with generalized hypohidrosis, which he had had since birth. He was born at term to nonconsanguineous parents, and underwent an emergent rescue of hyperpyrexia 3 days after his birth in a county hospital. Thereafter, he developed heat intolerance, palpitation, debilitation, facial hyperhidrosis, and trunk and limb anhidrosis following exercise, physical labor or exposure to a hot outdoor environment. These symptoms improved when he remained in areas of shade or washed in cold water. Since our district is a subtropical climate, he was accustomed to sleeping on the floor and cold

Approved by the Advisory Board and accepted for publication on 06.02.2014

Work performed at the Department of Dermatology, Affiliated Hospital of Guangdong Medical College - Guangdong, China.

Financial Support: This study was supported by the Doctoral Research Fund of the Affiliated Hospital of Guangdong Medical College (No. 1100/B010001). Conflict of Interest: None.

1 Affiliated Hospital of Guangdong Medical College -Guangdong, China.

(O2015 by Anais Brasileiros de Dermatologia 
water showering all year round. None of his parents, four siblings, two sons or daughter had hypohidrosis, while his medical history was unremarkable.

Examination revealed generalized dry skin with mild palmoplantar hyperkeratosis. The facies, hair and teeth and nails were normal but his beard and axillary hairs were sparse (Figure 1). In addition, his general health, intelligence, neurologic and ophthalmologic examination, and electrocardiography and abdominal ultrasonography were all normal. Furthermore, the complete blood count and urinalysis, general biochemical profile, thyroid function and cholinesterase levels were within normal limits.

Iodine-starch test showed a detectable perspiration (blue-black stain) on the forehead, cheeks, chin, axillae and palms, while no sweating was observed on the trunk or extremities (Figure 1). Histological examination of serial sections revealed that hair follicles were sparse and sweat glands were wholly absent from the dermis and hypodermis in the left forearm and right thigh, though some eccrine and apocrine sweat glands were present in the right axilla. S-100 was expressed in the nerve terminals surrounding the acini and ducts

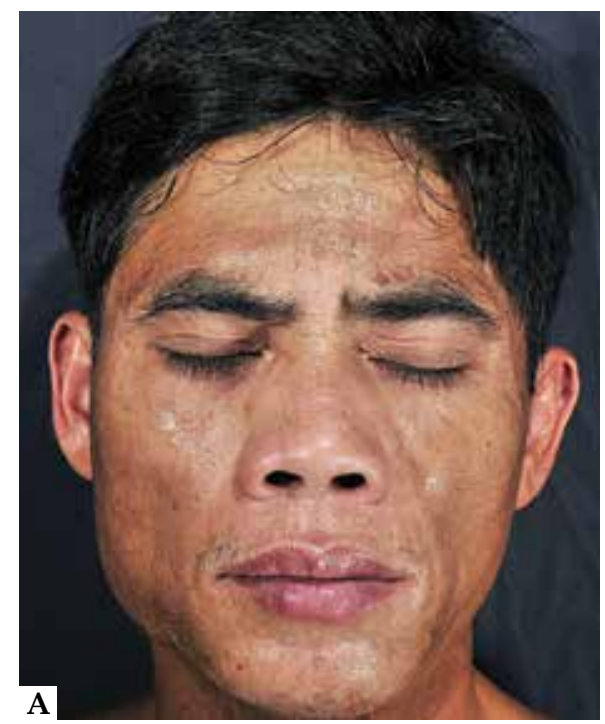

Figure 1:

Iodine-starch test:

A. Blue-black

stain on forehead,

cheeks and chin,

with a sparse

beard; B. Blue-

black stain on

both axillae, with

sparse axillary

hairs

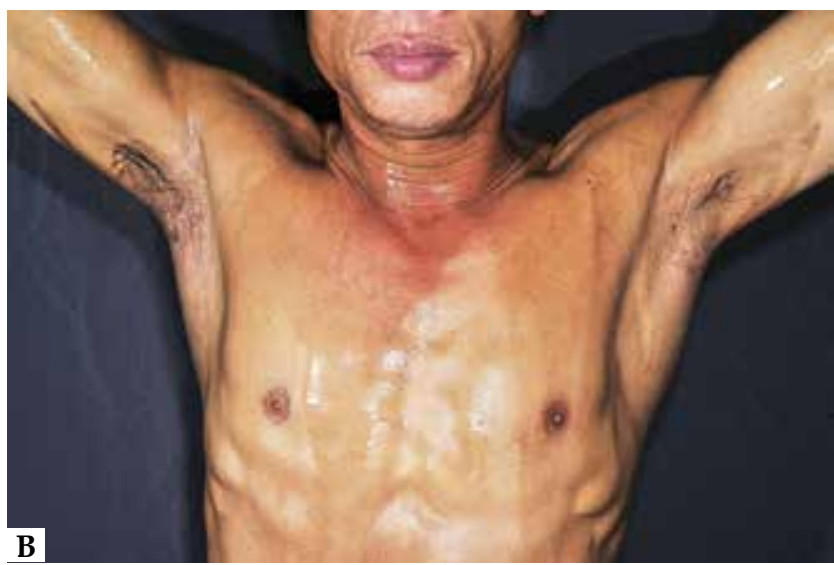

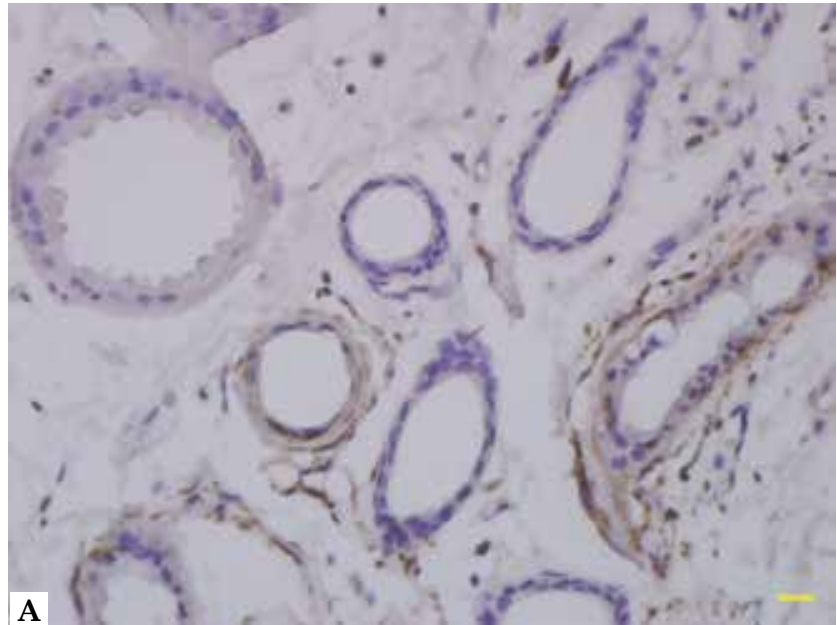

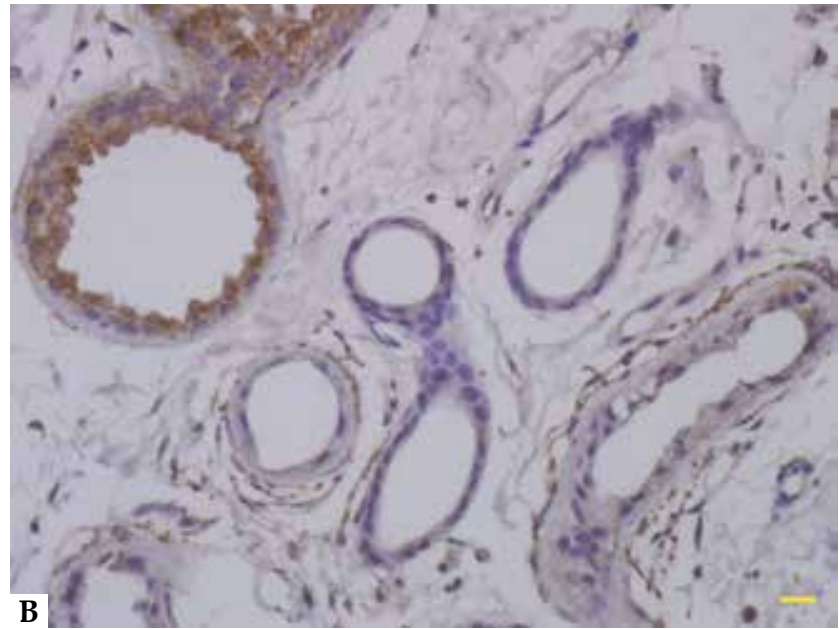

FIGURE 2: Immunohistochemical staining in the right axilla (bar=20 $\mu \mathrm{m})$ : A. S100 expression in the nerve terminals surrounding the acini and ducts of the eccrine sweat glands. B. PGP9.5 positivity in the acini of apocrine glands and nerve terminals surrounding the eccrine glands.

of the eccrine sweat glands, while PGP9.5 was positive in the acini of apocrine glands and the nerve terminals surrounding the eccrine glands in the right axilla (Figure 2).

\section{DISCUSSION}

Hypohidrosis involves reduced sweating in response to appropriate stimuli, and its etiology includes exogenous, dermatological (primary) and neuropathic (secondary) causes. Primary hypohidrosis may be congenital or acquired. ${ }^{7}$ Hypohidrotic ectodermal dysplasia (HED) is one of the most common genetic disorders characterized by defective development of teeth, hair, nails and eccrine sweat glands. Its inheritant models include $X$-linked recessive, and autosomal recessive and dominant patterns. ${ }^{8}$ However, congeni- 
tal idiopathic anhidrosis is very rare. Three siblings from an Iranian family, two boys and one girl, suffered from congenital idiopathic anhidrosis with complete absence of sweat glands. The consanguineous marriage and negative family history seemed to explain the autosomal recessive inheritance. ${ }^{2}$ In another Iranian family without consanguineous marriage, skin biopsy demonstrated the paucity of sweat glands in a brother and sister with simple hypohidrosis. ${ }^{5}$ Additionally, skin biopsies showed markedly atrophic sweat glands in a woman with congenital idiopathic anhidrosis, and she may have had HED selectively involving the eccrine glands. ${ }^{1,6}$

Our patient had sweated little since birth and suffered from no obvious abnormalities of other skin appendages, apart from his sparse beard and axillary hairs. The sweat test showed localized sweating on the face, axillae and palms. Serial sections showed complete absence of sweat glands in the left forearm and right thigh, though they were present in the right axilla. S-100 and PGP9.5 immunostaining revealed numerous nerve terminals surrounding the eccrine sweat glands in the axilla. These results suggest that his hypohidrosis is due to congenital absence other than denervation of the eccrine sweat glands in the large area.

Although his parents died of unknown diseases a few years ago, his siblings recalled that the parents had no defects concerning the teeth, hair or sweating. The starch-iodine test may help to differentiate $X$-linked and autosomal recessive HED in isolated patients, whilst playing a confirmative role in heterozygous carrier detection. ${ }^{9}$ His two elder sisters and daughter only underwent visual inspection, so the possibility of patchy and mild sweating disturbance was not excluded. Hence, this patient may represent the first case of congenital idiopathic hypohidrosis in China, in which the inheritant pattern and pathogenic genes require further evaluation.]

\section{REFERENCES}

1. Donadio V, Montagna P, Nolano M, Cortelli P, Misciali C, Pierangeli G, et al. Generalised anhidrosis: different lesion sites demonstrated by microneurography and skin biopsy. J Neurol Neurosurg Psychiatry. 2005;76:588-91.

2. Mahloudji M, Livingston KE. Familial and Congenital Simple Anhidrosis. Am J Dis Child. 1967;113:477-9.

3. Dann EJ, Berkman N. Chronic idiopathic anhydrosis--a rare cause of heat stroke. Postgrad Med J. 1992;68:750-2.

4. Thami GP, Kaur S, Kanwar AJ. Acquired idiopathic generalized anhidrosis: a rare cause of heat intolerance. Clin Exp Dermatol. 2003;28:262-4.

5. Frydman M, Cohen HA, Kauschansky A, Matoth Y. Familial simple hypohidrosis with abnormal palmar dermal ridges. Am J Med Genet. 1988;31:591-6.

6. Cevoli S, Pierangeli G, Magnifico F, Bonavina G, Barletta G, Candela C, et al. The circadian rhythm of body core temperature (CRT) is normal in patient with congenital generalized anhidrosis. Clin Auton Res. 2002;12:170-3.

7. Chia KY, Tey HL. Approach to hypohidrosis. J Eur Acad Dermatol Venereol. 2013;27:799-804.

8. Liu Y, Yu X, Wang L, Li C, Archacki S, Huang C, et al. Mutation p.Leu354Pro in EDA causes severe hypohidrotic ectodermal dysplasia in a Chinese family. Gene. 2012;491:246-50.

9. Cambiaghi S, Restano L, Pääkkönen K, Caputo R, Kere J. Clinical findings in mosaic carriers of hypohidrotic ectodermal dysplasia. Arch Dermatol. 2000;136:217-24.

\author{
MAILING ADDRESS: \\ Yi-Ming Fan \\ Department of Dermatology \\ Affiliated Hospital of Guangdong Medical College \\ Zhanjiang \\ 524001 Guangdong, China. \\ E-mail:ymfan1963@163.com
}

How to cite this article: Shi G, Zhu CY, Zhou Y, Yang YP, Fan YM. First case of congenital idiopathic hypohidrosis in China. An Bras Dermatol. 2015;90(5):731-3. 